\title{
A Study of the Errors in Locating Earthquakes due to the Geometry of the Taiwan Seismic Network
}

\author{
Yi-Ben Tsai ${ }^{1}$ and Hsin-Hung $\mathrm{Wu}^{1}$ \\ (Manuscript received 17 June 1996, in final form 12 December 1996)
}

\begin{abstract}
The Taiwan seismic network is operated by the Central Weather Bureau(CWB). It currently consists of 72 three-component stations and covers an area approximately $300 \mathrm{~km}$ long in the $\mathrm{N}-\mathrm{S}$ direction, and $100 \mathrm{~km}$ wide in the $E-W$ direction. The elongated geometry may cause a systematic bias in locating earthquakes in the Taiwan area. We conduct numerical experiments to investigate the possible errors caused by this geometry. We use this network to locate artificial earthquakes, and then study the location errors by comparing the computed with the true locations. We also use an artificial network to study the ability of the computer program routinely used by the CWB to locate earthquakes. The real data from the 15 December 1993 Tapu earthquake sequence are used to compare the quality of earthquake locations computed by the whole CWB network with that of those computed by excluding distant stations in the network.

It is found that the computer program itself will not cause the computed epicenters to shift along a narrow azimuthal range. More than $50 \%$ of artificial earthquakes with focal depths at $10 \mathrm{~km}$ or $20 \mathrm{~km}$ are located by the computer program at deeper depths under favorable situations. The errors caused by the program itself are less than $\mathbf{3 0 0}$ meters in epicenter locations, and less than $\mathbf{2 0 0}$ meters in focal depth determinations. The particular geometry of the CWB network will cause the computed epicenters to systematically shift along a narrow azimuthal range. The errors in epicenter locations are larger for deeper artificial earthquakes. The geometry of the CWB network will cause nearly all artificial earthquakes with focal depth $10 \mathrm{~km}$ or $20 \mathrm{~km}$ to be located at shallower depths. If there are errors in the plane-layered velocity model, the geometry of the CWB network still causes similar systematic errors in the epicenter locations. In this case, the shift of located epicenters is still confined to a narrow azimuthal range, but with a much larger distance. For artificial data, the distribution of epicenters computed by a sub-network, without distant stations (i.e. a subset of
\end{abstract}

${ }_{1}$ Institute of Geophysics, National Central University, Chung-Li, Taiwan, R.O.C. 
the CWB network), is similar to that computed by the whole CWB network. But those computed by far sub-networks (i.e. subsets of the CWB network) are significantly different from that computed by the whole CWB network. For real data, the epicenters computed by far sub-networks are also quite different from that computed by the whole CWB network. The quality of hypocenter locations computed by excluding distant stations is better than that computed by the whole CWB network. In summary, improvements in the quality of earthquake location can be achieved simply by not using distant stations to locate earthquakes in the Taiwan area.

\section{(Key words: Geometry, The CWB network, Artificial earthquake, Artificial network, Earthquake location)}

\section{INTRODUCTION}

The seismic network used by the Central Weather Bureau to locate earthquakes occurring in the Taiwan area has an elongated geometry spanning approximately $300 \mathrm{~km}$ in the N-S direction, and $100 \mathrm{~km}$ in the E-W direction. In this paper, we studied the possible bias in earthquake location caused by this geometry. The seismic activity in the Taiwan area is quite high, especially in the eastern offshore area where many earthquakes occur outside this network. Due to the network's elongated shape, for most earthquakes, the station coverage is not uniform around the epicenters. This particular geometry of the network will probably cause some errors in locating earthquakes in the Taiwan area

In order to accurately locate an earthquake, in addition to having a proper seismic velocity model and accurate arrival time data, we need to have a network with stations evenly distributed around the earthquake such that the azimuth gap(i.e. the largest difference in azimuths of any two neighboring stations of the network) is small(i.e. $<90$ ) in order to get well-constrained epicenter location. The network is also required to have a minimum distance(i.e. distance from the epicenter to the nearest station) shorter than the focal depth of the earthquake, and the observation of both direct and refracted waves in order to get a well-constrained focal depth determination(Lee and Stewart, 1981). For most earthquakes occurring in the Taiwan area, the network is not evenly distributed around them so it is inevitable that the geometry of this network causes errors in locating these earthquakes.

In this paper, we design simple numerical experiments using artificial data for the CWB network, and also an artificial network, to investigate the errors caused exclusively by the geometry of the CWB network, and the error inherently caused by the computer program itself (i.e. HYPON.FOR which is routinely used by the CWB for locating earthquakes). We also studied how the bias on earthquake location due to the geometry of CWB network is affected by an improper velocity model. Finally, we used real local earthquake data to study the quality of locations (i.e. epicenters and focal depths ) calculated respectively, by excluding distant stations, and by using the whole CWB network. There are observations of both direct and refracted waves made by near stations. It is expected that the negative effects of geometry for 
the near stations should be smaller than those for the distant stations.

\section{METHODS AND RESULTS}

In this paper we conducted three simple numerical experiments to assess possible location errors.

\subsection{Experiment 1:}

In this experiment, we try to calibrate the accuracy of the locating program itself.

We set up an artificial network (Figure 1) with stations evenly distributed on concentric circles having a common center. The radii of these circles are 5, 15, 35, 55, 100, 105 and 125 $\mathrm{km}$. There are eight stations on each circle. The artificial earthquake is located at the common center (Figure 1). Next, we artificially generate first $P$ and first $S$ arrival times to those sta-


Fig. 1. The artificial network. Its seismograph stations(solid triangles) are evenly distributed on seven circles with radii of $5,15,35,55,100,105$, and $125 \mathrm{~km}$. There are eight stations on each circle. The artificial earthquake(black dot) is located at the common center of these circles.
Fig. 2. Seismic velocity models : Model I and Model II. The velocities of layers 3 and 4 in Model II are $10 \%$ less than that of Model I. For other layers, both models have the same velocities. 
tions. The seismic velocity model used is the one routinely used by the CWB and is shown in Figure 2. Hereafter, this model will be referred to as Model I.

In order to eliminate their effect in earthquake location, there are no errors in the artificial data generated for this experiment. But in order to simulate realistic cases, we did randomly allow $20 \%$ of the stations not to receive P arrivals, and $40 \%$ of the stations not to receive $S$ arrivals. We set the focal depth of artificial earthquake at $10 \mathrm{~km}$ and $20 \mathrm{~km}$. For each depth, we generated 20 sets of artificial data, and then we used the same velocity model and different subsets of stations on this artificial network to locate the 20 artificial earthquakes. By comparing the calculated locations of focus with the true location, we can easily compute the errors in locating these earthquakes. Because the geometry of the network is completely symmetric, and there is no error in artificial data, and because we know the seismic velocity model exactly, so the errors in locations can be attributed solely to the locating program itself.

Figures 3(a) and 4(a) show that the mislocations of epicenter caused by the locating program itself are randomly distributed around the true location and the degrees of error are less than 300 meters. Figure 3(b) shows that the program tends to locate $10-\mathrm{km}$-depth earthquakes at shallower depths. In contrast, Figure 4(b) shows that the program tends to locate earthquakes of $20 \mathrm{~km}$ depth at deeper depths. When the farthest stations are at distances of at least

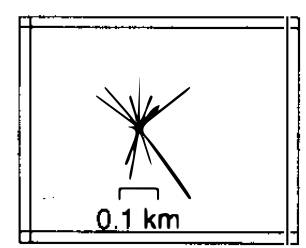

(a.1)

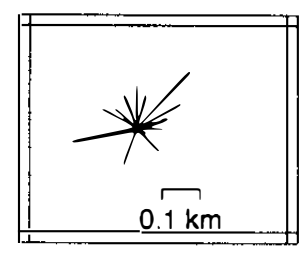

(a.3)

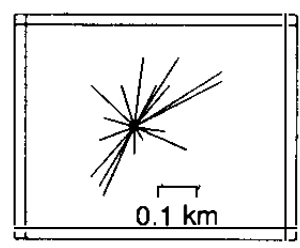

(a.1)

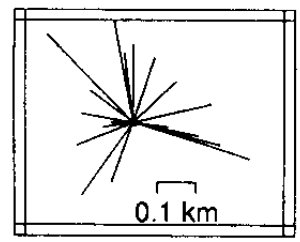

(a.3)

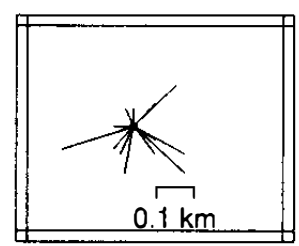

(a.2)

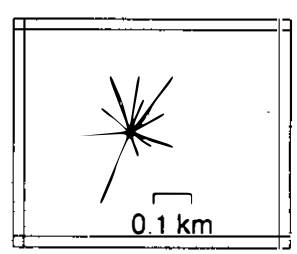

(a.4)

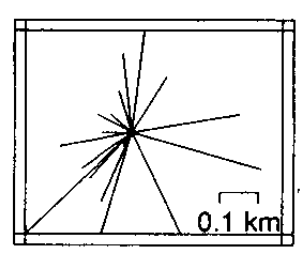

(a.2)

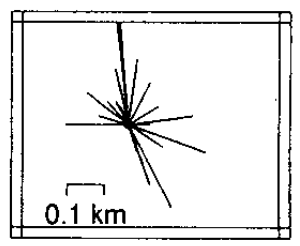

(a.4)
Fig. 3(a). Distribution of the computed epicenters(at the end of each line segment originating from the solid circle)of the 20 sets of data of each artificial earthquake(solid circle) at a true depth of $10 \mathrm{~km}$. (a.1) Epicenters computed by using 32 stations of the artificial network. These stations are on circles with radii of 5, 15, 35, and $55 \mathrm{~km}$. (a.2) Epicenters computed by using 40 stations on circles with radii of $5,15,35,55$, and $100 \mathrm{~km}$.(a.3) Epicenters computed by using 48 stations on circles with radii of $5,15,35,55,100$ and $105 \mathrm{~km}$.(a.4) Epicenters computed by using 56 stations on circles with radii of $5,15,35,55,100,105$ and $125 \mathrm{~km}$.

Fig. 4(a). Distribution of the computed epicenters. It is the same as in Figure 3(a). But the true focal depth is at $20 \mathrm{~km}$. 

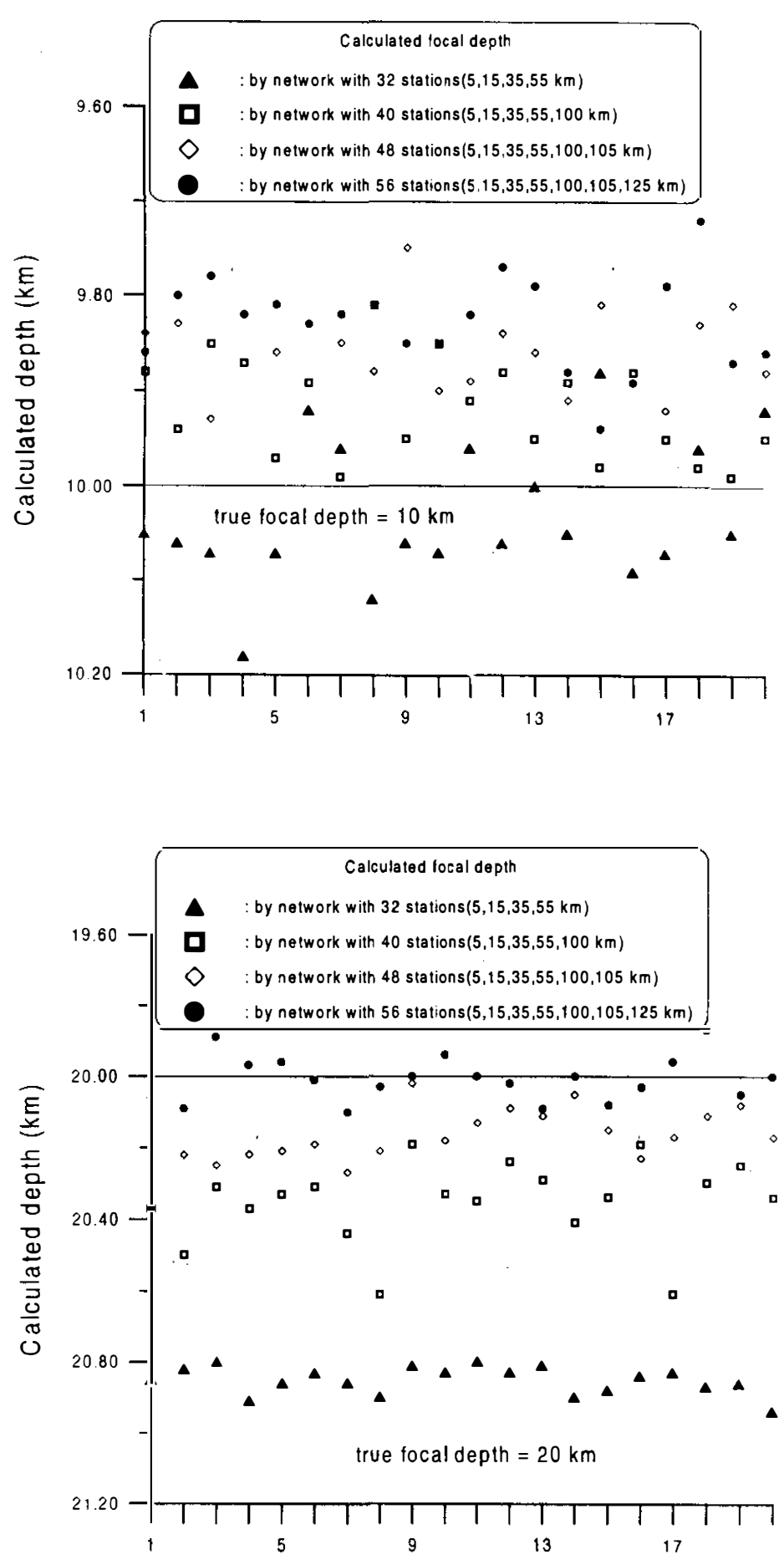

Fig. 3(b). Distribution of the computed focal depths of the 20 sets of artificial data of the artificial earthquake at a true depth of $10 \mathrm{~km}$. Different symbols are used for the focal depths calculated by networks with different number of stations.

Fig. 4(b). Distribution of the computed focal depths. As for Figure 3(b), but with the true focal depth at $20 \mathrm{~km}$.

five times the focal depths, the errors in focal depth determinations are less than 400 meters.

\subsection{Experiment 2 :}

In this experiment, we try to study the location errors caused by the geometry of the CWB network.

We choose five locations for the artificial earthquakes which are numbered 1 to 5 in Figure 5. We artificially generate first $P$ and first $S$ arrival times, again at depths of $10 \mathrm{~km}$ and 
$20 \mathrm{~km}$ for each artificial earthquake. For earthquake No. 2, we also generate artificial data at a depth of $50 \mathrm{~km}$. The seismic velocity model used is Model I. For each case, such as earthquake No. 1 at a depth of $10 \mathrm{~km}$, we generate 20 sets of artificial data, i.e. 20 artificial earthquakes to be located. There are no reading errors in these generated data. The only difference among these 20 sets of artificial data comes from the fact that we randomly allow $20 \%$ of the stations not to receive the first $P$ arrivals, and $40 \%$ of the stations not to receive the $S$ arrivals, to simulate realistic cases. Then, we use the same seismic velocity model (Model I) to locate these artificial earthquakes. After that, we compute the location errors by comparing the computed locations with the true location. The location errors must be due to the geometry of station distribution because there are no reading errors in the data, and we know the model exactly. In locating the events, for the same data set we use four different networks, namely the CWB network and its three sub-networks - the northern sub-network, central sub-network and southern sub-network, as shown in Figure 5.

Figures 6(a) and 7(a) show that the geomery of the CWB network can cause the mislocated epicenters to shift along a narrow azimuthal range, and that the location errors are larger for deeper events for all five artificial earthquakes, regardless of whether the artificial earthquake is inside or outside the network(see also Figure 8). Furthermore, Figures 6(b) and 7(b) show that the geometry also causes most of the artificial events with a focal depth of $10 \mathrm{~km}$ or $20 \mathrm{~km}$ to be located at shallower depths, but those No. 2 events with a focal depth of $50 \mathrm{~km}$ to be located at deeper depths(Figure 9).

Next, to locate those artificial earthquakes, we also use a different seismic velocity model which is derived from Model I and is named Model II, as shown in Figure 2. Then we can study how the location errors due to the geometry of the network are affected by the model error, by comparing the calculated locations with the true location. Figure 10 shows that if

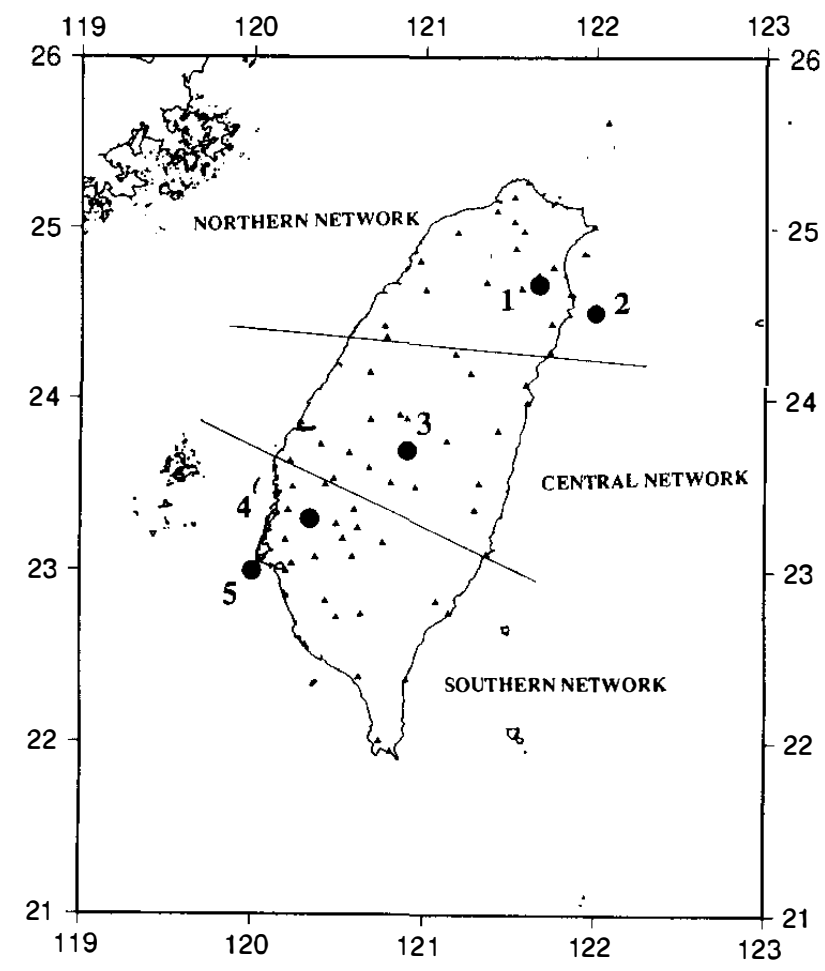

Fig.5. The CWB network (solid triangles)and the five artificial earthquakes(solid circles, numbered 1, 2, 3, 4, and 5). The two solid lines divide the CWB network into three smaller sub-networks, namely Northern, Central, and Southern sub-networks. 


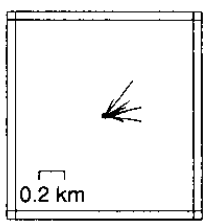

(a.1)

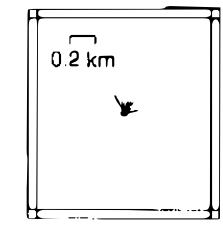

(a.3)

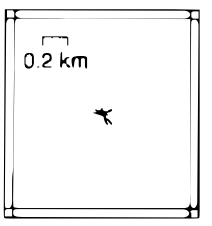

(a.4)

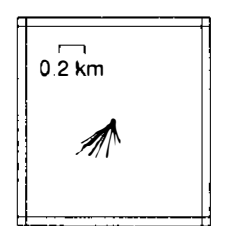

(a.2)

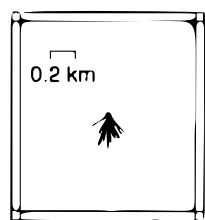

(a.5)

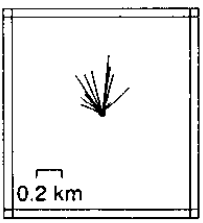

(a.1)

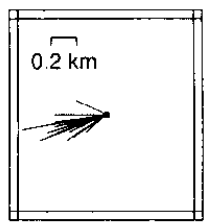

(a.2)

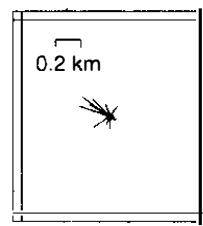

(a.3)

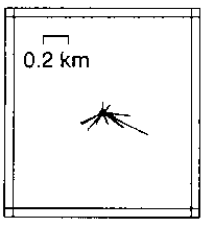

(a.4)

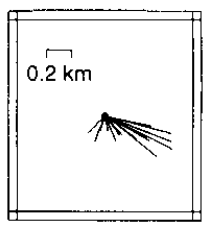

(a.5)
Fig. 6(a). Distribution of the computed epicenters(at the end of each line segment originating from the solid circle)of the 20 sets of data of each artificial earthquake (solid circle) at a depth of $10 \mathrm{~km}$. The whole CWB network is used : (a.1) is for artificial earthquake No. 1 ; (a.2) is for artificial earthquake No. 2;

Fig. 7(a). Distribution of the computed epicenters of five artificial earthquakes. As for Figure 6(a), except the true focal depth is at $20 \mathrm{~km}$. (a.3) is for artificial earthquake No.

3 ; (a.4) is for artificial earthquake No. 4 ; and (a.5) is for artificial earthquake No. 5 .

there is model error, the epicenters are still mislocated to positions within a narrow azimuthal range, but the degrees of errors are several times larger than those without model error.

\subsection{Experiment 3:}

In this experiment, we try to show with two sets of real earthquake data that in locating earthquakes, the exclusion of distant stations can give better results than the use of whole the CWB network.

\subsubsection{Case 1}

In this case, we choose five real earthquakes(Table 1) which occurred in 1994 near the location of artificial earthquake No. 2. We use the northern, central and southern sub-networks to relocate these five earthquakes with respect to the seismic velocity Model I . Then, we 

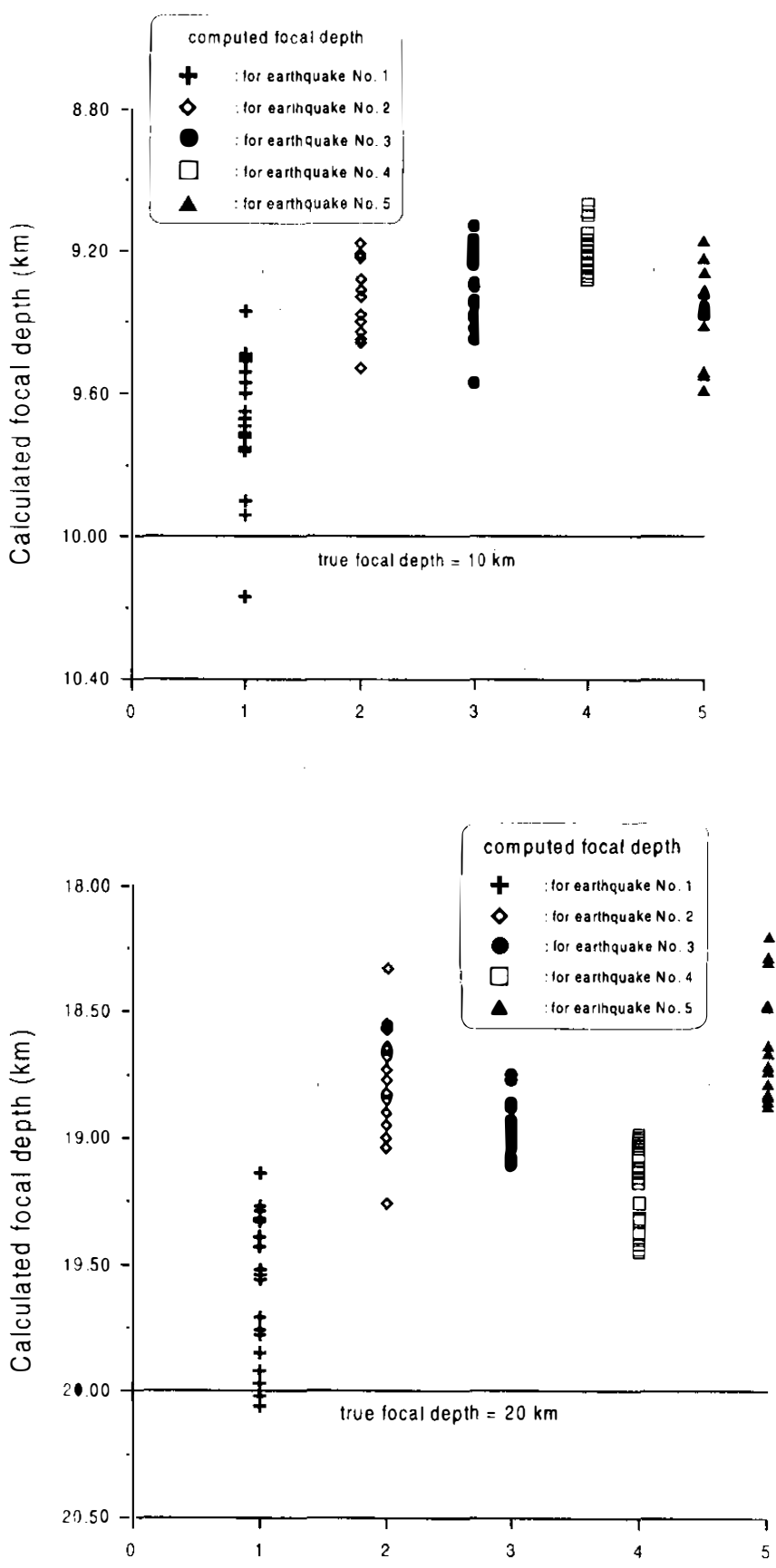

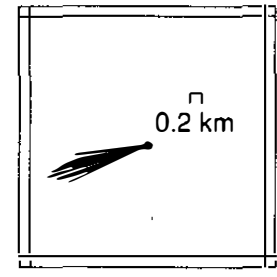

(a)

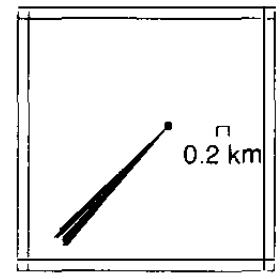

(c)

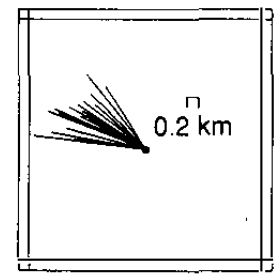

(b)

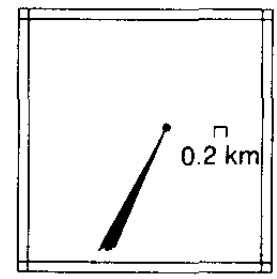

(d)
Fig. 6(b). Distribution of the computed focal depths of five artificial earthquakes. For each artificial earthquake, there are 20 sets of arrival time data, so there are 20 computed focal depths, and they are shown on a vertical column. The true depth is at $10 \mathrm{~km}$.
Fig. 7(b). Distribution of the computed focal depths of the five artificial earthquakes. As for Figure 6(b), except the true focal depth is at $20 \mathrm{~km}$.
Fig. 8. Distribution of computed epicenters(at the end of each line segment originating from the solid circle) of the 20 sets of data of artificial earthquake No. 2 (solid circle) at a true depth of $50 \mathrm{~km}$. (a) Epicenters computed by the whole CWB network (b) Epicenters computed by Northern sub-network (c) Epicenters computed by Central subnetwork (d) epicenters computed by Southern sub-network. 


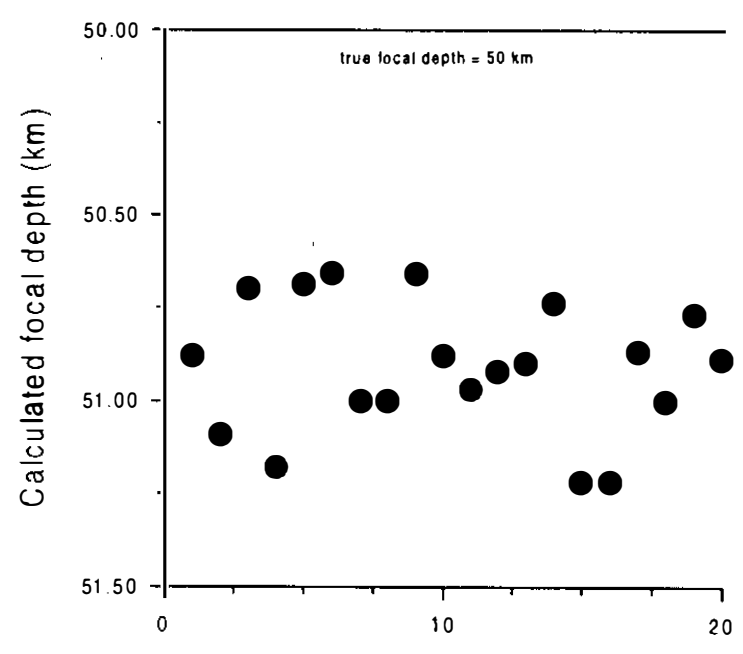

Fig. 10. Distribution of the computed epicenters. As for Figure 7(a), but the perturbed seismic velocity model (Model II) was used in locating these artificial earthquakes. So, in addition to the geometry of CWB network, there is model error to bias the computed epicenters.

Fig. 9. Distribution of computed focal depths of the 20 sets of data of artificial earthquake No. 2 (solid circle) at a true depth of $50 \mathrm{~km}$. Focal depths were computed by the whole CWB network.

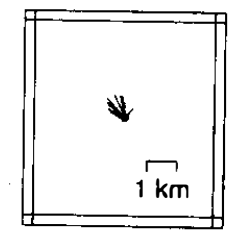

(a.1)

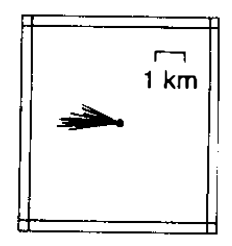

(a.2)

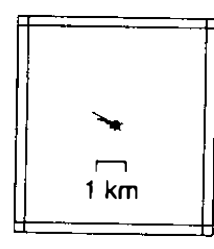

(a.3)

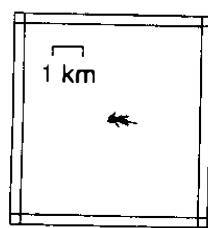

(a.4)

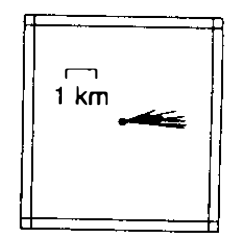

(a.5)

Table 1. The five real earthquakes located near artificial earthquake No. 2. The source parameters are calculated using the whole CWB network.

\begin{tabular}{lrrrrrrrrrrrr}
\hline \multicolumn{1}{l}{ No } & \multicolumn{4}{c}{ Origin time } & \multicolumn{4}{c}{ Epicenter } & Depth \\
& Y & M & D & H & M & \multicolumn{1}{c}{ S } & LAT(N) & LON(E) & $(\mathrm{km})$ \\
\hline 1 & 94 & 4 & 5 & 11 & 57 & 47.20 & 24 & 23.15 & 121 & 58.03 & 12.85 \\
2 & 94 & 4 & 29 & 3 & 35 & 43.15 & 24 & 23.60 & 122 & 00.01 & 15.13 \\
3 & 94 & 6 & 6 & 8 & 57 & 24.49 & 24 & 25.85 & 121 & 56.91 & 3.45 \\
4 & 94 & 6 & 17 & 21 & 36 & 6.37 & 24 & 26.59 & 121 & 54.73 & 2.06 \\
5 & 94 & 11 & 1 & 15 & 11 & 4.73 & 24 & 27.06 & 121 & 55.27 & 8.56 \\
\hline
\end{tabular}


compare the new locations with those previously computed using the whole CWB network. From the comparison, we can see the differences among the locations computed by these different sub-networks.

For the five real earthquakes located close to the position of artificial earthquake No. 2, the locations of their epicenters as calculated by the northern, central and southern sub-networks are different from those calculated by the CWB network. Figure 11 shows that the difference is quite large for the farthest network (i.e. the southern sub-network).

\subsubsection{Case 2}

In this case, we choose real data of the 15 December 1993 Tapu, Chiayi earthquake and its forty-six aftershocks. According to the report by the CWB (Seismological Bulletin, 1993), the earthquake and aftershocks were all shallower than $17 \mathrm{~km}$. The data files are provided by the CWB. Employing the same model as that routinely used by the CWB (i.e. Model I), we tried to relocate all the events of this earthquake sequence by using only subsets of stations on the CWB network.We used two subsets of stations; one with stations having epicentral distances of up to $100 \mathrm{~km}$, and the other with stations having epicentral distances of up to $55 \mathrm{~km}$. Then we can see the quality of the solutions calculated by the three sets of stations(i.e. those two subsets used by us and the set from the whole CWB network) by comparing the spatial distributions of calculated epicenters, and also by comparing the $\mathrm{P}$ wave travel time residuals computed as a function of epicentral distance. In relocating these earthquakes, we also consider the reading errors and weightings of the original data.

The quality of epicenter locations calculated by excluding distant stations is better than that computed by the whole CWB network. For the 15 December 1993 Tapu earthquake sequence, the epicenters calculated using stations having an epicentral distance of $100 \mathrm{~km}$ or less are more closely clustered than those computed by the whole CWB network, and when the epicenters are calculated using stations within $55 \mathrm{~km}$ (see Figure 12), the results are even better. Figure 13 shows that the station $\mathrm{P}$ travel time residuals as a function of epicentral distance are closer to zero for smaller epicentral distances, and become larger for larger epicentral distances. The $\mathrm{P}$ residuals calculated by stations within $100 \mathrm{~km}$ are smaller than those calculated using the whole CWB network. Figure 14 shows that of the focal depths computed by stations with epicentral distances within $55 \mathrm{~km}$, more than $80 \%$ are deeper than those calculated using the whole CWB network. It seems that the whole CWB network has a tendency to locate these earthquakes at shallower depths.

\section{DISCUSSION}

In testing the accuracy of the locating program, HYPON.FOR, no data error or model error are assumed. Furthermore, the artificial network is completely symmetrical around the artificial earthquake. Therefore the situation for locating earthquakes is nearly optimal: the azimuthal gap is small(i.e. 45), the smallest epicentral distance is less than the focal depth, and there are both direct and refracted waves among the first arrivals. Thus, except for the locating program, all factors which can bias the calculated locations are eliminated. The mislocated 


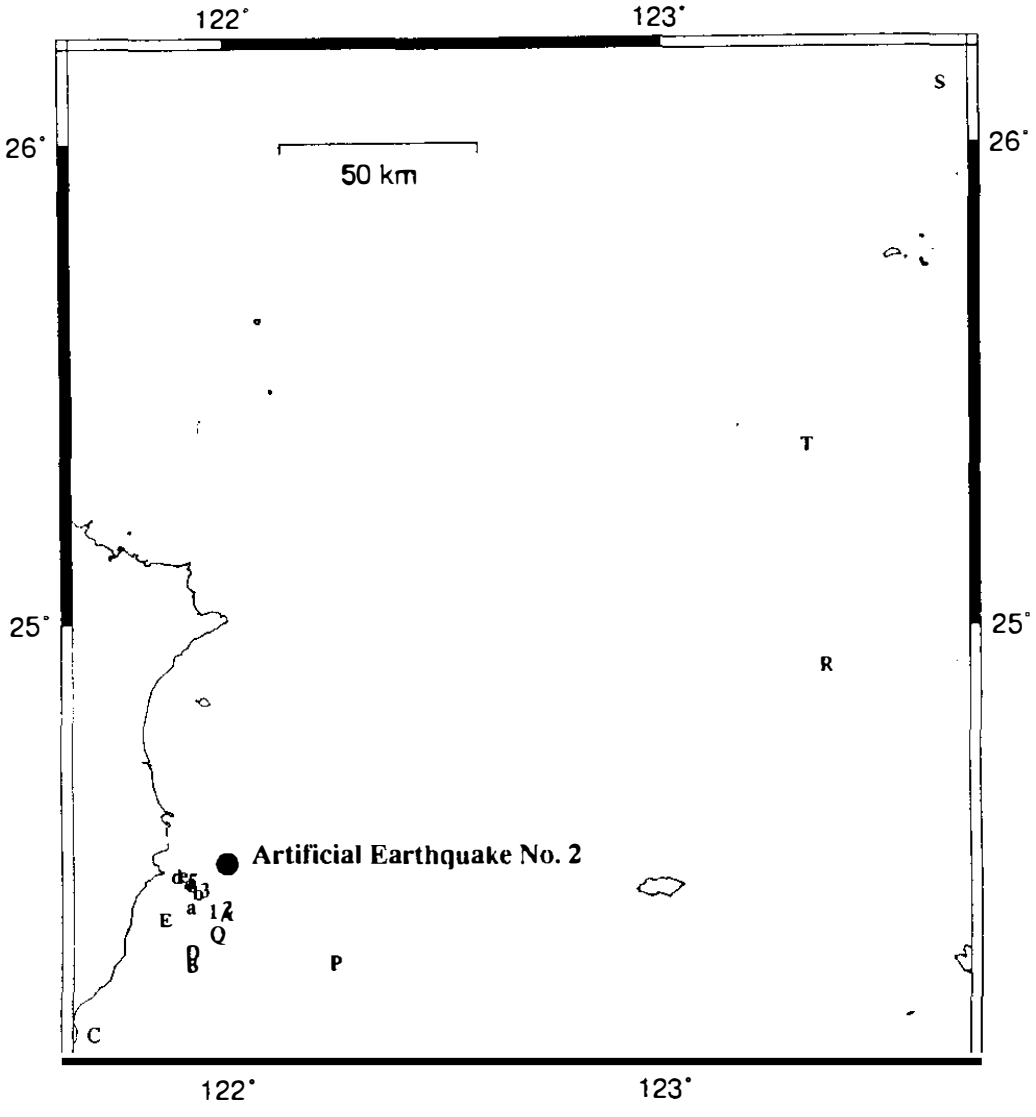

Fig. 12. Distribution of epicenters of 15 December 1993 Tapu earthquake(solid square) and its 46 aftershocks(black dots). (a) Epicenters computed by the whole CWB network (b) Epicenters computed by stations with epicentral distance 100 km (c) Epicenters computed by stations with epicentral distance $55 \mathrm{~km}$.
Fig. 11. Distribution of computed epicenters of those five real earthquakes located near to artificial earthquake No. 2(black solid circle). The epicenters computed by the whole CWB network are represented by $1,2,3,4,5$. The corresponding epicenters computed by the Northern sub-network are a,b,c,d,e, by the Central subnetwork are A,B,C,D,E, and by the Southern sub-network are $\mathrm{P}, \mathrm{Q}, \mathrm{R}, \mathrm{S}, \mathrm{T}$.

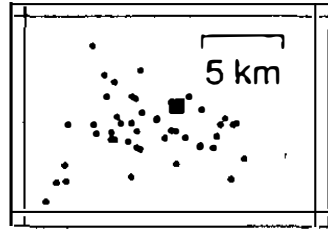

(a)

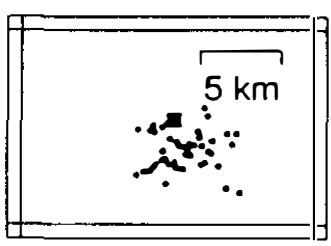

(b)

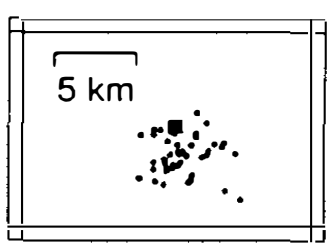

(c)

epicenters are randomly distributed in azimuths around the true epicenter, as shown in Figures 3(a) and 4(a). The degree of errors in epicenter determination is less than 300 meters. So, there is no evidence that the program itself will bias the calculated epicenters to shift along a finite azimuthal range.

The computed focal depths are shown in Figures 3(b) and 4(b). In most cases, earthquakes with a focal depth at $10 \mathrm{~km}$, and $20 \mathrm{~km}$, are located at shallower depths (Figure 3(b)) and 


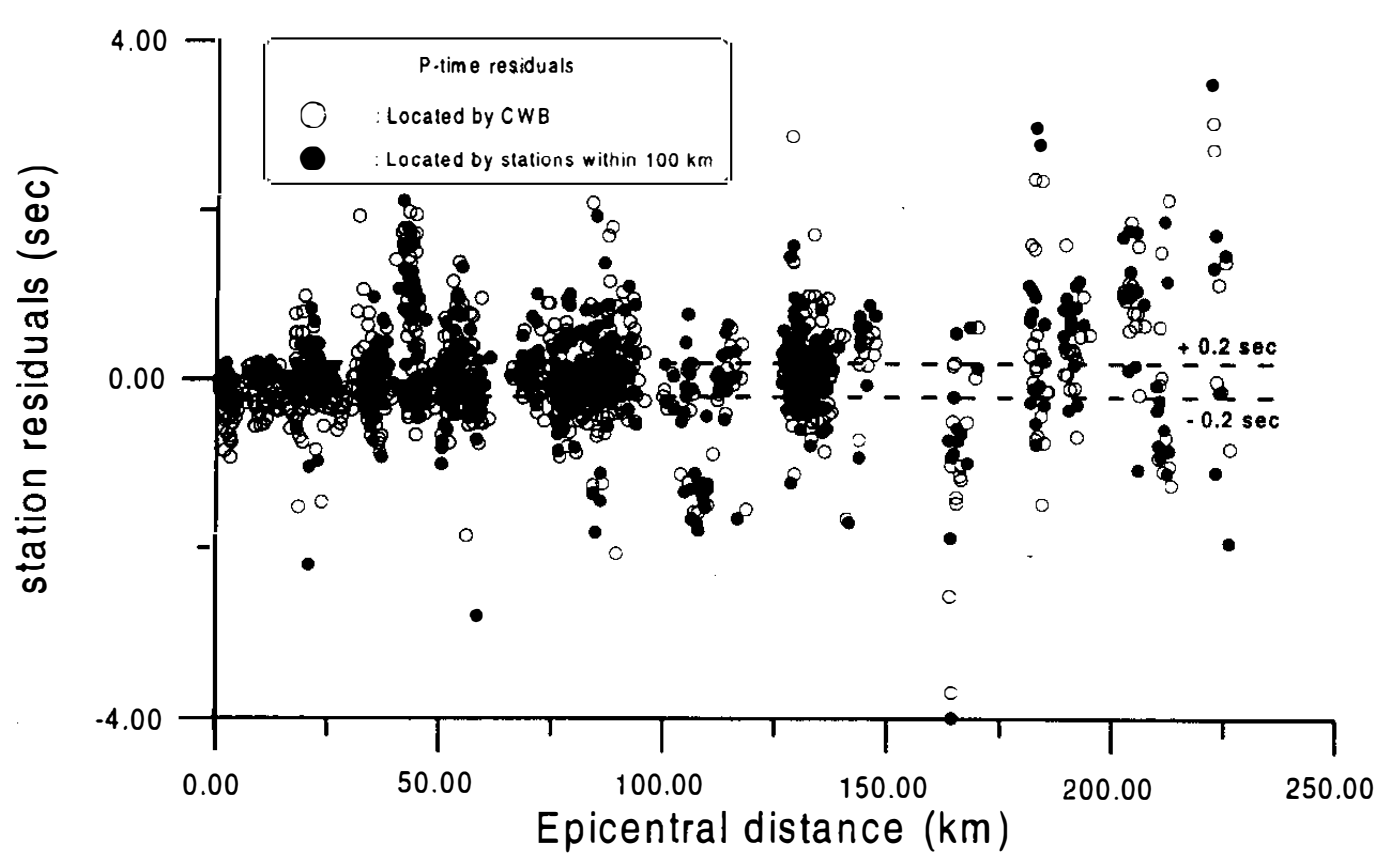

Fig. 13. Distribution of $\mathrm{P}$ wave travel time residuals as a function of epicentral distance. Open circles represent residuals determined by the whole CWB network, and solid circles represent residuals determined by stations with epicentral distance $100 \mathrm{~km}$. The two dashed lines represent residuals of +0.2 and -0.2 sec.

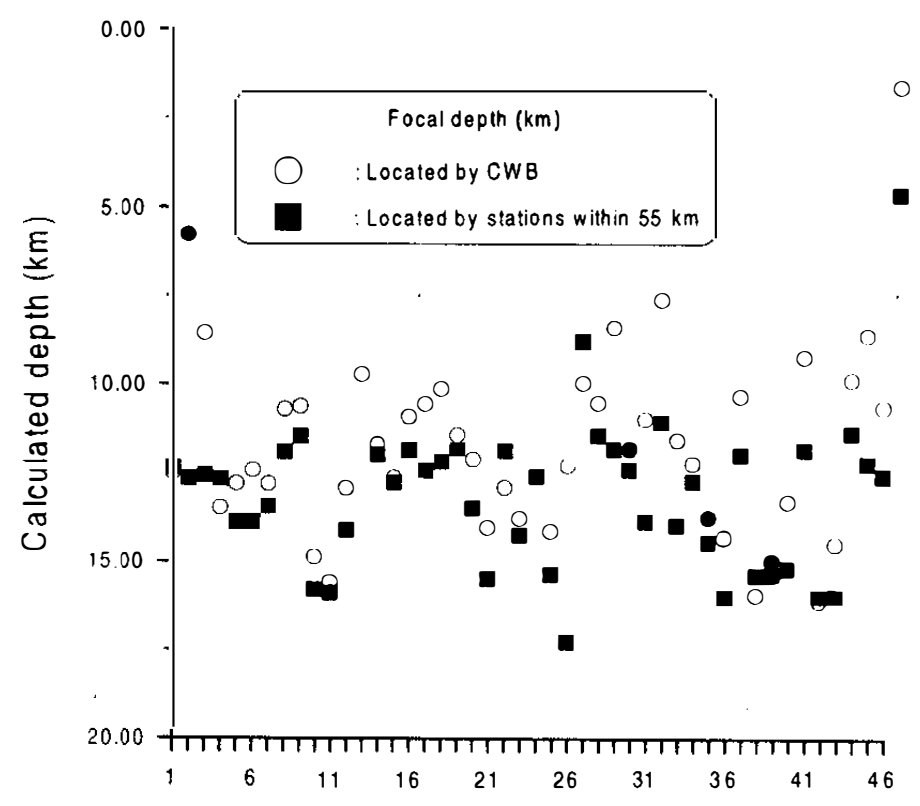

Fig. 14. Distribution of focal depths of 1993 Tapu earthquake and its 46 aftershocks. Open circles represent focal depths computed by the whole CWB network. Solid squares represent focal depths computed by stations with epicentral distance $55 \mathrm{~km}$.

deeper depths (Figure 4(b)), respectively. The degree of errors in focal depth determination is smaller than 400 meters for most cases. As far as the quality of focal depth determination is concerned, the network with 32 stations (epicentral distance $55 \mathrm{~km}$ ) is the best for earthquakes 
with a focal depth of $10 \mathrm{~km}$ (Figure 3(b)), and the network with 56 stations (epicentral distance $125 \mathrm{~km}$ ) is the best for earthquakes with a focal depth of $20 \mathrm{~km}$ (Figure 4(b)). Under these favorable conditions, the program locate more than $50 \%$ of the earthquakes at greater depths.

In studying the effect of the geometry of the CWB network, no model error and no data error are assumed. Also, from the results of Experiment 1 we know that locating program itself will not cause the epicenters to be mislocated along a narrow azimuthal range. The distributions of epicenters computed are shown in Figures 6(a) and 7(a). It is easy to see that the geometry of the CWB network will cause the epicenters to be mislocated along a narrow azimuthal range for all five artificial earthquakes, with the effect being bigger for deeper earthquakes. Figures 6(a) and 7(a) also show that the effect of the geometry of the CWB network is smaller for earthquakes which are located within the network and are more evenly surrounded by seismograph stations. So, the mislocations of the epicenters of events No. 1, 3 and 4 are smaller, especially for event No. 3 and 4. The stations around these two earthquakes are quite evenly distributed(Figure 5).

Lai(1996) studied the differences between the epicenters computed by a joint network consisting of the CWB network and four OBS stations (No. 1, 2, 3, and 4 in Figure 16) and that computed by only the CWB network. Her results are quite consistent with ours. Figure 16 shows that her earthquakes No. 80,82,17, 22, and 16 are within the joint network. Therefore, the epicenters computed by this joint network are more likely to be better than those computed by the CWB network alone. So, if we take the former epicenters as the true ones, then this figure shows that the epicenters computed by the CWB network are systematically shifted west. This is consistent with our results as shown in (a.2) of Figures 6(a), 7(a), and 8 which show that the computed epicenters of artificial earthquake No. 2 are indeed shifted west. In Figure 17, the earthquakes shown are outside (i.e. south of)the joint network. However, for these earthquakes the distribution of joint network should be better than that of the CWB network. Therefore, the epicenters computed by this joint network should be better and closer to the true locations. So, the figure again shows that the epicenters computed by only the CWB network are mislocated to positions within a narrow azimuthal range. This is also consistent with our results as shown in (a.2) of Figures 6(a), 7(a), and 8.

The above results suggest that an effective remedy for the mislocation of offshore epicenters is to deploy some OBS stations that will make station distribution more even around earthquakes. On land, we need to have denser station distribution.

An interesting fact to note is that the poorly constrained direction is not always in the direction perpendicular to the trend of the CWB network as was often generally supposed ((a.3) and (a.5) of Figure 6(a) and (a.1) of Figure 7(a)). It quite probably depends on the relative location of an earthquake with respect to the distribution of stations.

Except for a few data sets of earthquake No. 1, all artificial earthquakes with focal depths at $10 \mathrm{~km}$ or $20 \mathrm{~km}$ are located by the CWB network at shallower depths (Figures 6(b) and 7(b)). It seems that the CWB network has a tendency to do this because the locating program will locate more than $50 \%$ of earthquakes with focal depths of $10 \mathrm{~km}$ or $20 \mathrm{~km}$ at deeper depths. For the Tapu earthquake sequence, whole CWB network consistently locates more than $80 \%$ of the earthquakes in the sequence at shallower depths than those calculated using 
only the stations with an epicentral distance of less than $55 \mathrm{~km}$. The events of earthquake No. 2 at $50 \mathrm{~km}$ depth are located by CWB network to deeper depths(Figure 9).

The distribution of epicenters calculated using a perturbed velocity model (Model II) to locate the five artificial earthquakes is also shifted along a narrow azimuthal range(Figure 10). But the degree of errors in epicenter location is magnified by 4 5 times. This means that under the influence of model error (assuming no reading errors in data), the geometry of the CWB network is dominating the bias on the direction of mislocation in epicenter determinations. It seems that, for a plane-layered model, the model uncertainty has a minor effect on the direction of mislocation of epicenters, but the geometry of station distribution has a major effect. On the other hand, the degree of error in the computed epicenter location is increased several times by the model error.

In testing the effect of the geometry of the CWB network (experiment 2), the locations of epicenters calculated by the near network are similar to those computed by the whole CWB network. For example, for artificial earthquake No. 1, the near network is the northern subnetwork and Figures 15(a) and 15(b) show that the distributions of computed epicenters are quite similar. In contrast, solutions obtained by the far sub-network differ more from that obtained by the whole CWB network (Figure 15).

For those five real shallow earthquakes located near to artificial earthquake No. 2, the locations of epicenters calculated by the far network are also quite different from that computed by the whole CWB network: So, it is not advantageous to include the far stations of CWB network in locating shallow earthquakes. Owing to the complicated structure of Taiwan area, the particular geometry of the CWB network will make the seismic waves travel through several tectonic provinces before arriving at far stations. The arrival times of these waves will have larger errors and make the calculated epicenters less accurate, because the solutions obtained by the whole CWB network need to fit all data well, even those with larger errors.

To support the above argument, we choose the 15 December 1993 Tapu earthquake sequence, and relocate their hypocenters by excluding far stations and using real data (p-times, s-times, their reading errors and weightings). The distributions of epicenters are shown in Figure 12. Evidently, the distribution of epicenters calculated by excluding far stations is more closely clustered as often expected for an earthquake sequence. This conclusion is also supported by the distribution of $\mathrm{P}$ wave travel time residuals as a function of epicentral distance (Figure 13). Our result shows that the solutions obtained by using only the near stations fit the data of the near stations better than other solutions (i.e. closer to zero residuals), with the fit getting worse for data for far stations (i.e. larger residuals). The possible reasons for the near stations having better hypocenter determinations are as follows:

(1) The data reading error is smaller for near stations, because the signal to noise ratio is larger for these stations.

(2) The error due to incorrect velocity model is smaller for the near stations because less crustal structure complications are involved.

(3)The bias due to the particular geometry of the CWB network is reduced.

In summary, the geometry of the CWB network can cause the computed epicenters to shift along a narrow azimuthal range and the effect is larger for earthquakes outside the network or earthquakes with deeper focal depths. If there is model error, the geometry still domi- 


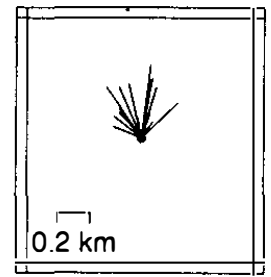

(a)

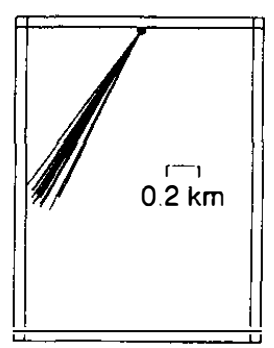

(c)

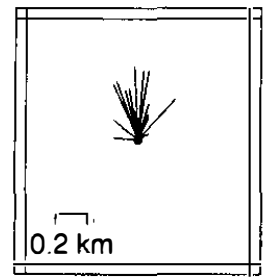

(b)

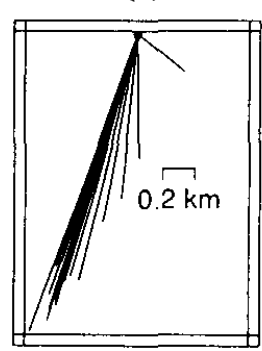

(d)
Fig. 15. Distribution of computed epicenters( the end of each line segment originating from the solid circle)of the 20 sets of data of artificial earthquake No. 1 (solid circle) at a true depth of $20 \mathrm{~km}$. (a) Epicenters computed by the whole CWB network (b)Epicenters computed by the Northern sub-network (c) Epicenters computed by the Central subnetwork (d) epicenters computed by the Southern sub-network.
Fig. 16. The distribution of epicenters. $\boldsymbol{\Delta}$ : epicenters computed by the joint network, $\diamond$ : epicenters computed by the CWB network, + : the OBS stations, $\square$ : the CWB stations. (modified from Figure 13[a] from Lai[1996])
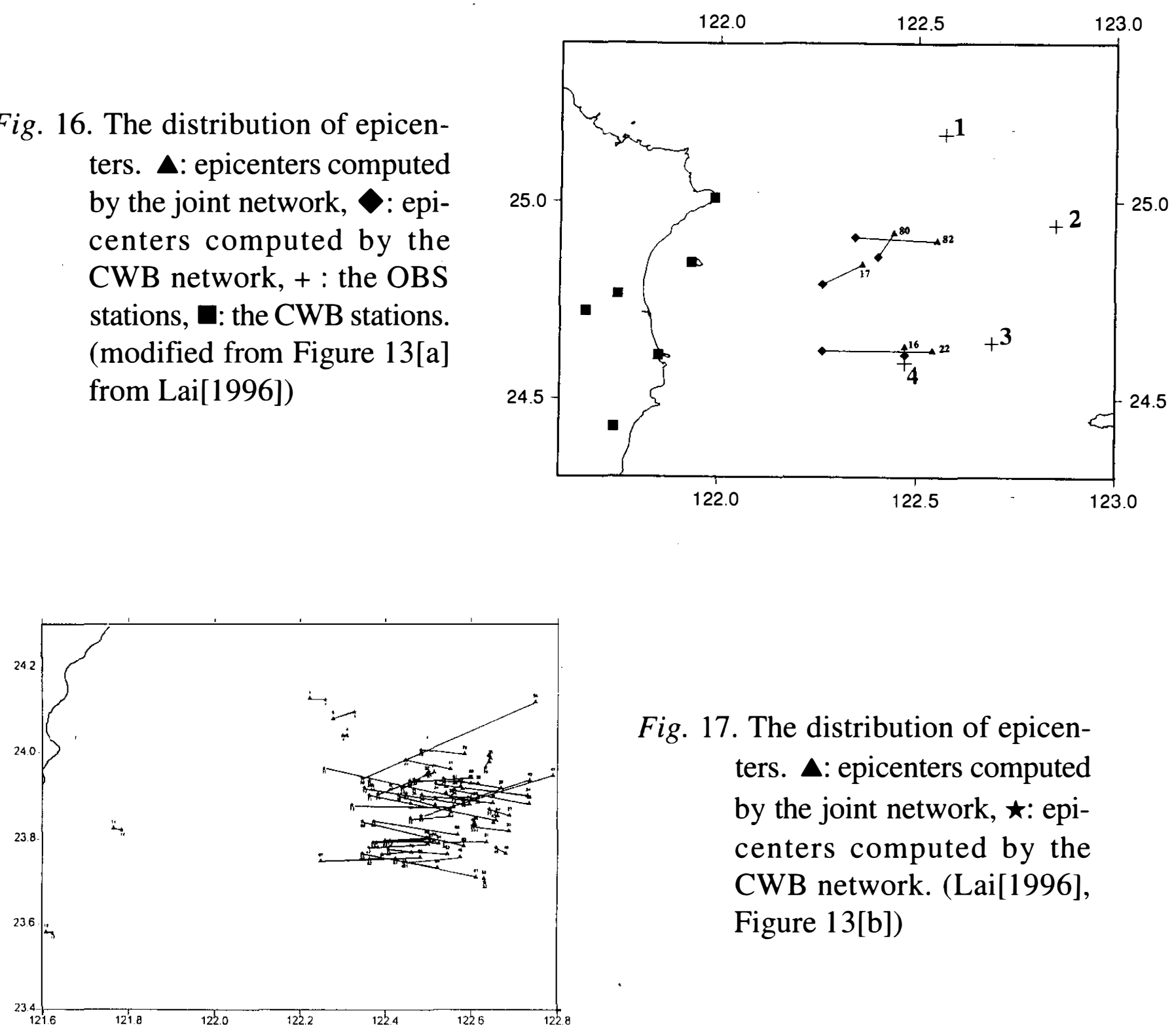

Fig. 17. The distribution of epicenters. $\boldsymbol{\Delta}$ : epicenters computed by the joint network, $\star$ : epicenters computed by the CWB network. (Lai[1996], Figure 13[b]) 
nates the bias in the azimuths of mislocated epicenters. In conclusion, it is advantageous not to use far stations to locate earthquakes in the Taiwan area. This is particularly true for earthquakes inside the seismic network.

\section{CONCLUSIONS}

The accuracy of the computer program routinely used for locating earthquakes by the CWB is excellent. The geometry of the CWB network tends to cause the located epicenters to shift along a narrow azimuthal range, and has a tendency to locate earthquakes with focal depth $10 \mathrm{~km}$ or $20 \mathrm{~km}$ at shallower depths. The quality of hypocenter solutions computed by excluding far stations (i.e. stations with epicentral distances greater than several times the focal depth of the located earthquake) appears to be better than that calculated by the whole CWB network. In summary, it is not necessarily advantageous in practice to include far stations to locate earthquakes in Taiwan area. This finding has positive implications for rapid earthquake location for early warning purposes because the waiting time for far station arrival times can be excluded..

Acknowledgments The authors thank Dr. W. H. K. Lee for valuable comments and discussion. We also thank the CWB for providing the locating program and real earthquake data.

\section{REFERENCES}

Central Weather Bureau(1993): SEISMOLOGICAL BULLETIN, Vol.40, No.4, October to December, $117 \mathrm{pp}$.

Lee, W. H. K. and J. C. Lahr, (1975). HYPO71(REVISED): A computer program for determining hypocenter, magnitude, and first motion pattern of local earthquakes, U. S. Geol. Surv. Open-File Report 75-311, 114pp.

Lee, W. H. K. and S. W. Stewart, (1981): Principles and Applications of Microearthquake Networks. Advances in Geophysics supplement 2, 292pp.

Lai, M. C.,(1996): Earthquake observations and its implications in the northeast offshore area of Taiwan. Masters thesis, National Taiwan Oceanic University, 77pp. 